\title{
Rational approximations to linear forms in values of $G$-functions
}

\author{
by \\ Makoto Nagata (Tokyo)
}

Introduction. In 1929, C. L. Siegel [8] defined two classes of functions in transcendence theory. One is the class of $E$-functions (for the definition, see [7]) and the other is the class of $G$-functions (see $\S 2$ below). Proofs of the algebraic independence for values of $E$-functions and transcendence measures were first originated by Siegel, and developed by A. B. Shidlovskii and his students. On the contrary, for $G$-functions, Siegel only gave a program for the proofs of the linear independence of their values. After a while, A. I. Galochkin [6] proved some irrationality measures. He used the method of Padé approximations of the first kind. His proofs require an assumption, which is called the Galochkin condition. This assumption appeared in E. Bombieri's paper [3] in another form, but it was not removed from the irrationality statements. In 1985, D. V. Chudnovsky and G. V. Chudnovsky overcame this difficulty [4, Theorem III]. They proved that the Galochkin condition holds a priori. They also used a refined method of classical Padé approximations. Furthermore, they obtained some measures for values of $G$-functions $[4$, Theorem I] without the Galochkin condition.

To explain the present state of research on the irrationality measures for values of $G$-functions, let us introduce the following (unproved) statement:

Statement 0.1. Let $f_{1}(x), \ldots, f_{m}(x)$ be $G$-functions. Assume that $f_{1}(x), \ldots, f_{m}(x)$ are linearly independent over $\mathbb{Q}(x)$ and that the 1 -column matrix

$$
\bar{f}:={ }^{t}\left(f_{1}(x), \ldots, f_{m}(x)\right)
$$

is a solution of a first order linear differential equation

$$
\frac{d}{d x} \bar{f}=A \bar{f},
$$

where $A \in M_{m \times m}(\mathbb{Q}(x))$. Let $r=a / b \in \mathbb{Q}, r \neq 0$, and $0<\varepsilon<1 / 2$. Then there exist a constant $C_{1}$, depending on $f_{1}(x), \ldots, f_{m}(x), \varepsilon$, and a constant 
$C_{2}$, depending on $f_{1}(x), \ldots, f_{m}(x), \varepsilon, r$, such that if

$$
|b|^{\varepsilon}>C_{1}|a|^{m(m-1+\varepsilon)},
$$

then for arbitrary integers $H_{1}, \ldots, H_{n}$ such that $H:=\max _{i=1, \ldots, m}\left|H_{i}\right|$ $>C_{2}$,

$$
\left|\sum_{i=1}^{m} H_{i} f_{i}(r)\right|>\frac{H^{1-\varepsilon}}{\prod_{i=1}^{m} \max \left(\left|H_{i}\right|, 1\right)}
$$

D. V. Chudnovsky and G. V. Chudnovsky proved a weaker form of Statement 0.1 [4, Theorem I], with (0.1.1) replaced by

$$
\left|\sum_{i=1}^{m} H_{i} f_{i}(r)\right|>H^{1-m-\varepsilon} .
$$

We state our result.

TheOREM 0.2. Let $f_{i}(x)(i=1, \ldots, n)$ be a non-zero solution of a scalar linear differential equation of order $m_{i}$ over $\mathbb{Q}(x)$ :

$$
\left(\frac{d}{d x}\right)^{m_{i}} f_{i}(x)+a_{i, m_{i}-1}(x)\left(\frac{d}{d x}\right)^{m_{i}-1} f_{i}(x)+\ldots+a_{i, 0}(x) f_{i}(x)=0,
$$

where $a_{i, j}(x) \in \mathbb{Q}(x)\left(i=1, \ldots, n ; j=0, \ldots, m_{i}-1\right)$. Put $f_{i}^{(j)}(x):=$ $(d / d x)^{j} f_{i}(x)$ and $m:=\sum_{i=1}^{n} m_{i}$. Assume $f_{i}^{(j)}(x)(i=1, \ldots, n ; j=0, \ldots$ $\left.\ldots, m_{i}-1\right)$ are $G$-functions linearly independent over $\mathbb{Q}(x)$. Let $r=a / b \in$ $\mathbb{Q}, r \neq 0$, and let $0<\varepsilon_{0}<1 / 2$ be a fixed real number. Then there exist an effective constant $C_{3}$, depending only on $f_{i}^{(j)}(x)(i=1, \ldots, n$; $\left.j=0, \ldots, m_{i}-1\right), \varepsilon_{0}, m$, and an effective constant $C_{4}$, depending only on $f_{i}^{(j)}(x)\left(i=1, \ldots, n ; j=0, \ldots, m_{i}-1\right), \varepsilon_{0}, m, r$, such that if

$$
|b|^{\varepsilon_{0}}>C_{3}|a|^{2 m(m+1)},
$$

then for arbitrary integers $H_{i}^{(j)}\left(i=1, \ldots, n ; j=0, \ldots, m_{i}-1\right)$ such that $H:=\max _{i=1, \ldots, n}\left(\left|H_{i}\right|\right)>C_{4}$ and $H_{i}:=\max _{j=0, \ldots, m_{i}-1}\left(\left|H_{i}^{(j)}\right|, 1\right)$,

$$
\left|\sum_{i=1}^{n} \sum_{j=0}^{m_{i}-1} H_{i}^{(j)} f_{i}^{(j)}(r)\right|>\frac{H^{1-\varepsilon_{0}}}{H_{1}^{m_{1}} \cdots H_{n}^{m_{n}}} .
$$

As compared with the methods of [4, Theorem I], our method estimates simultaneously several systems of $G$-functions. D. and G. Chudnovsky introduced the method of graded Padé approximations, and announced that Statement 0.1 can be proved by their methods ([4, Theorem V $]$ ).

We use classical Padé approximations and obtain the best possible measures for some special cases $\left(m_{1}=\ldots=m_{n}=1\right.$ in Theorem 0.2). Statement 0.1 is still unproved, at least by the method of classical Padé approximations. 
This paper is organized as follows.

In Section 1 we prove some properties of our Padé approximations. We follow more or less the method developed by Shidlovskiu for $E$-functions [7, Chapter 3] until Lemma 1.8 which improves on Shidlovskiı's counterpart: the matrix that appears in Shidlovskil's method is too big to provide good bounds for $G$-functions. It is important to deal with a matrix of minimal size and maximum rank for the final estimates to depend only on the $H_{i}$ 's.

Section 2 contains the estimations of linear forms in Padé polynomials. The Galochkin condition appears as the $(G, C)$-property. (See Lemma 2.5 below.) We use there the result of [4]. Furthermore, at the end of the section we obtain a matrix of integers which has good approximations.

Section 3 contains the proof of our result. The sketch of the proof is as follows: Using the estimates of the first two sections, we obtain an inequality involving the data, the degrees of the Padé polynomials and their orders at 0 . This inequality provides the bound in our result provided that these degrees and orders satisfy two conditions. The condition on their orders is transformed into another one on the degrees of the Padé polynomials. We reduce them to conditions on $\varepsilon$ and $H$, that is, we prove the existence of the Padé polynomials with these conditions for any $0<\varepsilon<1 / 2$ and for any large $H$. We conclude our estimations by these Padé polynomials.

1. Padé approximations. Let $\Lambda$ be the set of indices

$$
\Lambda:=\left\{(i, j) \mid i=1, \ldots, n ; j=1, \ldots, m_{i}\right\}
$$

and write

$$
m:=\sum_{i=1}^{n} m_{i}
$$

We consider the power series $f_{(i, j)}=f_{(i, j)}(x) \in \mathbb{Q}[[x]]((i, j) \in \Lambda)$.

For parameters $D, D_{i}, T \in \mathbb{Z}(i=1, \ldots, n)$, consider $P_{(i, j)}=P_{(i, j)}(x) \in$ $\mathbb{Q}[x]$ with

$$
\begin{gathered}
\operatorname{deg} P_{(i, j)} \leq D, \\
\operatorname{ord}_{x=0} P_{(i, j)} \geq D-D_{i} \quad((i, j) \in \Lambda),
\end{gathered}
$$

such that

$$
R:=\sum_{(i, j) \in \Lambda} P_{(i, j)} f_{(i, j)} \in \mathbb{Q}[[x]]
$$

satisfies

$$
\operatorname{ord}_{x=0} R \geq T
$$

and $R \neq 0$. 
These polynomials $P_{(i, j)}$ always exist under a condition on $T$. See Remark 1.2 below. They are not uniquely defined.

Definitions 1.1. We call $P_{(i, j)}$ Padé approximants and $R$ remainder function in the Padé approximation problem for $f_{(i, j)}$ with parameters $\left(T, D, D_{i}\right)$.

Henceforth we write

$$
D:=\max _{i=1, \ldots, n} D_{i}
$$

and we assume that

$$
D_{i} \geq 2 \varepsilon D
$$

and

$$
T \geq\left[\sum_{i=1}^{n} m_{i} D_{i}-\varepsilon D\right]
$$

for a sufficiently small $\varepsilon>0$. Here $[\alpha]$ means the integer part of $\alpha$.

Remark 1.2. The number of the coefficients of $P_{(i, j)}$ for $(i, j) \in \Lambda$ is at most $\sum_{i=1}^{n}\left(m_{i} D_{i}+1\right)$. If

$$
T<\sum_{i=1}^{n}\left(m_{i} D_{i}+1\right)
$$

there exists a non-trivial solution of the Padé approximation problem with parameters $\left(T, D, D_{i}\right)$.

Notations 1.3. Define an $m$-tuple of power series by

$$
\bar{f}:={ }^{t}\left(f_{(1,1)}, \ldots, f_{\left(1, m_{1}\right)}, f_{(2,1)}, \ldots, f_{\left(n, m_{n}\right)}\right) \in \mathbb{Q}[[x]]^{m} .
$$

We fix $A_{(i)} \in M_{m_{i}}(\mathbb{Q}(x))$, the set of $m_{i} \times m_{i}$ matrices, for $i=1, \ldots, n$ and

$$
A:=\left(\begin{array}{ccc}
A_{(1)} & & 0 \\
& \ddots & \\
0 & & A_{(n)}
\end{array}\right) \in M_{m}(\mathbb{Q}(x)) .
$$

Consider the differential equation

$$
\frac{d}{d x} \bar{f}=A \bar{f},
$$

which means that

$$
\frac{d}{d x} \bar{f}_{i}=A_{(i)} \bar{f}_{i} \quad(i=1, \ldots, n),
$$

where $\bar{f}_{i}:={ }^{t}\left(f_{(i, 1)}, \ldots, f_{\left(i, m_{i}\right)}\right) \in \mathbb{Q}[[x]]^{m_{i}}$.

Furthermore, define a sequence of $m$-tuples of polynomials by

$$
\bar{p}^{[0]}:={ }^{t}\left(P_{(1,1)}, \ldots, P_{\left(1, m_{1}\right)}, P_{(2,1)}, \ldots, P_{\left(n, m_{n}\right)}\right) \in \mathbb{Q}[x]^{m}
$$


and recursively for $k=1,2, \ldots$,

$$
\bar{p}^{[k]}:=u\left(\frac{d}{d x}+{ }^{t} A\right) \bar{p}^{[k-1]}:={ }^{t}\left(P_{(1,1)}^{[k]}, \ldots, P_{\left(n, m_{n}\right)}^{[k]}\right) \in \mathbb{Q}[x]^{m},
$$

where

$$
u:=u(x)=\text { the lowest common denominator }
$$

$$
\text { of the entries of } A \in \mathbb{Z}[x] \text {. }
$$

In other words, one defines sequences of $m_{i}$-tuples by

$$
\bar{p}_{i}^{[0]}:={ }^{t}\left(P_{(i, 1)}, \ldots, P_{\left(i, m_{i}\right)}\right) \in \mathbb{Q}[x]^{m_{i}} \quad(i=1, \ldots, n)
$$

and for $k=1,2, \ldots$,

$$
\bar{p}_{i}^{[k]}:=u\left(\frac{d}{d x}+{ }^{t} A\right) \bar{p}_{i}^{[k-1]} \in \mathbb{Q}[x]^{m_{i}} \quad(i=1, \ldots, n) ;
$$

then it is clear that $\bar{p}^{[k]}$ are the tuples of all entries of $\bar{p}_{i}^{[k]}(i=1, \ldots, n)$.

Then we put

$$
R^{[0]}:={ }^{t} \bar{p}^{[0]} \bar{f}=\sum_{i=1}^{n}{ }^{t} \bar{p}_{i}^{[0]} \bar{f}_{i} \in \mathbb{Q}[[x]]
$$

and for $k=1,2, \ldots$,

$$
R^{[k]}:=\left(u \frac{d}{d x}\right)^{k} R^{[0]} \in \mathbb{Q}[[x]] .
$$

By elementary calculations, one has

$$
\begin{aligned}
R^{[k]} & =\left(u \frac{d}{d x}\right)^{k} R^{[0]}=\left(u \frac{d}{d x}\right)^{k-1}\left(u\left(\frac{d}{d x} t^{t} \bar{p}^{[0]}+{ }^{t} \bar{p}^{[0]} A\right) \bar{f}\right) \\
& =\left(u \frac{d}{d x}\right)^{k-1}\left(u\left(\left(\frac{d}{d x}+{ }^{t} A\right) \bar{p}^{[0]}\right) \bar{f}\right) \\
& =\left(u \frac{d}{d x}\right)^{k-1}\left({ }^{t} \bar{p}^{[1]}\right) \bar{f}=\ldots={ }^{t} \bar{p}^{[k]} \bar{f}
\end{aligned}
$$

Write

$$
R_{i}^{[0]}:={ }^{t} \bar{p}_{i}^{[0]} \bar{f}_{i} \in \mathbb{Q}[[x]]
$$

and for $k=1,2, \ldots$,

$$
R_{i}^{[k]}:=\left(u \frac{d}{d x}\right)^{k} R_{i}^{[0]} \in \mathbb{Q}[[x]] \quad(i=1, \ldots, n) .
$$

Similarly to (1.3.12) one finds that for $k=0,1,2, \ldots$,

$$
R_{i}^{[k]}={ }^{t} \bar{p}_{i}^{[k]} \bar{f}_{i} \quad(i=1, \ldots, n) .
$$


Finally, put

$$
s:=\max (\operatorname{deg} u-1, \operatorname{deg} u A) .
$$

Lemma 1.4. Let $P_{(i, j)}$ be Padé approximants and $R$ be the remainder function in the Padé approximation problem for $f_{(i, j)}$ with parameters $\left(T, D, D_{i}\right)$. Then $P_{(i, j)}^{[k]}$ are Padé approximants and $R^{[k]}$ is the remainder function with parameters $\left(T-k, D+k s, D_{i}+k s+k\right)$ for $k=0,1, \ldots, T$.

Proof. It is enough to show that

$$
\begin{gathered}
P_{(i, j)}^{[k]} \in \mathbb{Q}[x], \\
\operatorname{deg} P_{(i, j)}^{[k]} \leq D+k s, \\
\operatorname{ord}_{x=0} P_{(i, j)}^{[k]} \geq D-D_{i}-k, \\
\operatorname{ord}_{x=0} R^{[k]} \geq T-k .
\end{gathered}
$$

This follows immediately from the definitions.

We formulate Shidlovski1̌'s lemma [7, Chapter 3, Lemma 8] as the following proposition, which is implicit in his proof.

Proposition 1.5. Suppose that $f_{(i, j)}((i, j) \in \Lambda)$ are linearly independent over $\mathbb{C}(x)$ and satisfy the differential equation (1.3.3). For arbitrary polynomials $P_{(i, j)}^{[0]} \in \mathbb{C}(x)$ with

$$
\operatorname{deg} P_{(i, j)}^{[0]} \leq D \quad((i, j) \in \Lambda)
$$

let

$$
R^{[0]}=\sum_{(i, j) \in \Lambda} P_{(i, j)}^{[0]} f_{(i, j)} \in \mathbb{C}[[x]]
$$

and let

$$
R^{[k]}=\left(u \frac{d}{d x}\right)^{k} R^{[0]}=\sum_{(i, j) \in \Lambda} P_{(i, j)}^{[k]} f_{(i, j)}
$$

for $k=1,2, \ldots$ Furthermore, let

$$
l=\operatorname{rank}\left(P_{(i, j)}^{[k]}\right)_{(i, j) \in \Lambda}^{\substack{0 \leq k<m \\(i, j)}} .
$$

Then there exists a set $\Lambda_{1} \subset \Lambda$ with $\# \Lambda_{1}\left(=\right.$ the cardinality of $\left.\Lambda_{1}\right)=l$ such that

$$
\Delta=\operatorname{det}\left(P_{(i, j)}^{[k]}\right)_{(i, j) \in \Lambda_{1}}^{0 \leq k<l} \neq 0 .
$$

Finally, denote by $\Delta_{\mu, \nu}$ the $(\mu, \nu)$ th cofactor of $\Delta$. Then there exists a positive constant $\tau_{0}$, depending only on $f_{(i, j)}$, and there exist, for $\mu=1, \ldots, n$, 
power series $F_{\mu} \in \mathbb{C}[[x]]$ and polynomials $G_{\mu} \in \mathbb{C}(x)$, which depend only on $f_{(i, j)}$, such that

$$
\operatorname{ord}_{x=0} F_{\mu} \leq \tau_{0}
$$

and

$$
\Delta F_{\mu}=G_{\mu} \sum_{\nu=1}^{n} \Delta_{\mu, \nu} R^{[\nu-1]} .
$$

Pr o of. The first assertion is proved in [7, Chapter 3, Lemma 6]. (1.5.2) and (1.5.3) are implicit in the proof of [7, Chapter 3, Lemma 8].

For the sake of convenience, we often write for $i=1, \ldots, n$,

$$
D_{i, j}:=D_{i} \quad\left(j=1, \ldots, m_{i}\right) .
$$

LEMMA 1.6. Under the assumptions of Lemma 1.4, suppose that $\bar{f}$ satisfies the differential equation (1.3.3) and that the entries of $\bar{f}$ are linearly independent over $\mathbb{C}(x)$. Let

$$
l=\operatorname{rank}\left(P_{(i, j)}^{[k]}\right)_{\substack {i, j) \in \Lambda \\
\begin{subarray}{c}{0 \leq k<m \\
\text {. }{ i , j ) \in \Lambda \\
\begin{subarray} { c } { 0 \leq k < m \\
\text { . } } }\end{subarray}}
$$

Then there exist a positive constant $\gamma_{0}$, depending only on $\bar{f}$, and a set $\Lambda_{2} \subset \Lambda$ with $\# \Lambda_{2}=l$ such that

$$
\operatorname{ord}_{x=0} R \leq\left(\sum_{(i, j) \in \Lambda_{2}} D_{i, j}\right)+\gamma_{0} .
$$

Proof. We use Proposition 1.5. Let $\Delta$ be a minor of maximal rank of $\left(P_{(i, j)}^{[k]}\right)_{\substack{0, j<\in \Lambda \\ 0}, \Delta_{\mu, \nu}}$ the $(\mu, \nu)$ th cofactor of $\Delta$ and $\delta_{\mu, \nu}$ the $(\mu, \nu)$ th entry of $\Delta$. Each index $\mu$ corresponds to a double index $(i, j)$ such that $\delta_{\mu, *}=$ $P_{(i, j)}^{[*]}$; we denote this double index $(i, j)$ by $\lambda_{\mu}$. Let $\Lambda_{1}$ be a subset of $\Lambda$ which satisfies (1.5.1). From Lemma 1.4, one has

$$
\operatorname{deg} \Delta \leq l D+\frac{l(l-1)}{2} s
$$

and

$$
\operatorname{ord}_{x=0} \Delta_{\mu, \nu} \geq(l-1) D-\sum_{(i, j) \in \Lambda_{1} \backslash\left\{\lambda_{\mu}\right\}} D_{i, j}-l(l-1) / 2,
$$

or a fortiori

$$
\operatorname{ord}_{x=0} \Delta F_{\mu} \leq l D+\frac{l(l-1)}{2} s+\tau_{0}
$$

and there exists $\mu_{0}$ such that

$$
\operatorname{ord}_{x=0} G_{\mu} \sum_{k=1}^{l} \Delta_{\mu, k} R^{[k-1]} \geq \operatorname{ord}_{x=0} \sum_{k=1}^{l} \Delta_{\mu, k} R^{[k-1]}
$$




$$
\geq \operatorname{ord}_{x=0} R-l+(l-1) D-\sum_{(i, j) \in \Lambda_{1} \backslash\left\{\lambda_{\mu_{0}}\right\}} D_{i, j}-l(l-1) / 2
$$

for $\mu=1, \ldots, l$. Hence we have

$$
\operatorname{ord}_{x=0} R \leq D+\sum_{(i, j) \in \Lambda_{1} \backslash\left\{\lambda_{\mu_{0}}\right\}} D_{i, j}+\frac{l(l+1)}{2}(s+1)+l+\tau_{0}
$$

from (1.5.3). From (1.1.7), the index $\mu_{0}$ can be picked in such a way that $D_{\lambda_{\mu_{0}}}=D$. For this $\mu_{0},(1.6 .7)$ yields inequality (1.6.2) with $\# \Lambda_{2}=l$.

LEMMA 1.7. Under the assumptions of Lemma 1.6, for any $\varepsilon>0$ and for any $D>\widetilde{D}_{1}$, if

$$
\operatorname{ord}_{x=0} R \geq\left[\sum_{i=1}^{n} m_{i} D_{i}-\varepsilon D\right]
$$

then

$$
\operatorname{rank}\left(P_{(i, j)}^{[k]}\right)_{(i, j) \in \Lambda}^{0 \leq k<m}=m,
$$

where $\widetilde{D}_{1}$ is a positive constant depending only on $\bar{f}$ and $\varepsilon$. Furthermore, let

$$
\Delta:=\operatorname{det}\left(P_{(i, j)}^{[k]}\right)_{(i, j) \in \Lambda}^{0 \leq k<m}
$$

Then

$$
\operatorname{ord}_{x=0} \Delta \geq(m-\varepsilon) D-\frac{m(m+1)}{2}-1-\max _{(i, j) \in \Lambda}\left(\operatorname{ord}_{x=0} f_{(i, j)}\right)
$$

and

$$
\operatorname{ord}_{x=0} \Delta \leq m D+\frac{m(m-1)}{2} s .
$$

Pr o of. For the first assertion (1.7.2), we assume that

$$
l:=\operatorname{rank}\left(P_{(i, j)}^{[k]}\right)_{(i, j) \in \Lambda}^{0 \leq k<m}<m .
$$

Then there exists $\Lambda_{2} \subset \Lambda$ with $\# \Lambda_{2}=l$ such that

$$
\operatorname{ord}_{x=0} R \leq\left(\sum_{(i, j) \in \Lambda_{2}} D_{i, j}\right)+\gamma_{0} .
$$

This contradicts (1.1.8) for $D>\left(\gamma_{0}+1\right) / \varepsilon$, showing (1.7.2).

Let $\Delta_{\mu, \nu}$ be the $(\mu, \nu)$ th cofactor of $\Delta$. We have

$$
\Delta f_{(i, j)}=\sum_{\mu=1}^{m} \Delta_{\mu, \nu} R^{[\mu-1]},
$$


where $\nu=\sum_{k=1}^{i-1} m_{k}+j$. From Lemma 1.4, one finds for $\nu=0,1, \ldots, m-1$,

$$
\begin{aligned}
\operatorname{ord}_{x} & =0 \sum_{\mu=1}^{m} \Delta_{\mu, \nu} R^{[\mu-1]} \\
& \geq\left[\sum_{i=1}^{n} m_{i} D_{i}-\varepsilon D\right]-m+\sum_{(i, j) \in \Lambda}^{\nu}\left(D-D_{i, j}\right)-m(m-1) / 2,
\end{aligned}
$$

where $\sum_{(i, j) \in \Lambda}^{\nu}$ means that $(i, j)$ runs in $\Lambda$ with $\sum_{k=1}^{i-1} m_{k}+j \neq \nu$. From (1.1.7), for $(i, j)$ with $D=D_{i, j}$, we have

$$
\begin{aligned}
\operatorname{ord}_{x=0} \Delta f_{(i, j)} \geq & {\left[\sum_{i=1}^{n} m_{i} D_{i}-\varepsilon D\right]+\sum_{(i, j) \in \Lambda}^{\nu}\left(D-D_{i}\right) } \\
& -m(m-1) / 2-m-D-D_{i, j} \\
\geq & m D-\varepsilon D-m(m-1) / 2-m-1,
\end{aligned}
$$

or weakly,

(1.7.11) $\operatorname{ord}_{x=0} \Delta \geq m D-\varepsilon D-m(m-1) / 2-1-\max _{(i, j) \in \Lambda}\left(\operatorname{ord}_{x=0} f_{(i, j)}\right)$.

The inequality (1.7.5) follows immediately from Lemma 1.4.

LEMMA 1.8. Under the assumptions of Lemma 1.7, for any number $\xi$ with $\xi u(\xi) \neq 0$ and any $D>\widetilde{D}_{2}$,

$$
\operatorname{rank}\left(P_{(i, j)}^{[k]}(\xi)\right)_{\substack{i, j) \in \Lambda \\ 0 \leq k<m}}=m,
$$

where $\widetilde{D}_{2}$ is a positive constant depending only on $\bar{f}, u, \varepsilon$.

Pro of. We follow the procedure of [7, Chapter 3, Lemma 10]. Let $\Delta$ be as in (1.7.3) and

$$
\vartheta:=\operatorname{ord}_{x=\xi} \Delta .
$$

Suppose that ${ }^{t}\left(y_{1,1}, \ldots, y_{n, m_{n}}\right)$ is a solution of the differential equation (1.3.3) and use the notations (1.3.10) and (1.3.11). Then we have

$$
\Delta y_{i, j}=\sum_{\mu=1}^{m} \Delta_{\mu, \nu} R^{[\mu-1]},
$$

where $\Delta_{\mu, \nu}$ is the $(\mu, \nu)$ th cofactor of $\Delta$ and $\nu=\sum_{k=1}^{i-1} m_{k}+j$.

Operating $\left(u \frac{d}{d x}\right)$ on the identity (1.8.3), one finds

$$
u\left(\frac{d}{d x} \Delta\right) y_{i, j}+\Delta L_{1, i, j, 0}=\sum_{\mu=1}^{m+1} M_{1, \mu, \nu} R^{[\mu-1]}
$$

with $L_{1, i, j, 0}$ a linear combination of $y_{1,1}, \ldots, y_{n, m_{n}}$ over $\mathbb{C}(x)$ and $M_{1, \mu, \nu} \in$ $\mathbb{C}[x]$. 
Repeating this process $\vartheta$ times yields

$$
\left(u^{\vartheta}\left(\frac{d}{d x}\right)^{\vartheta} \Delta\right) y_{i, j}+\sum_{k=0}^{\vartheta-1}\left(\left(\frac{d}{d x}\right)^{k} \Delta\right) L_{\vartheta, i, j, k}=\sum_{\mu=1}^{m+\vartheta} M_{\vartheta, \mu, \nu} R^{[\mu-1]}
$$

with $L_{\vartheta, i, j, k}$ a linear combination of $y_{1,1}, \ldots, y_{n, m_{n}}$ over $\mathbb{C}(x)$ and $M_{\vartheta, \mu, \nu} \in$ $\mathbb{C}[x]$.

Since

$$
\left.\left(\left(\frac{d}{d x}\right)^{k} \Delta\right)\right|_{x=\xi}=0 \quad(k \leq \vartheta-1) \quad \text { and }\left.\quad\left(\left(\frac{d}{d x}\right)^{\vartheta} \Delta\right)\right|_{x=\xi} \neq 0,
$$

we obtain

$$
\begin{aligned}
& \text { 8.6) }\left.\left(\left(u^{\vartheta}\left(\frac{d}{d x}\right)^{\vartheta} \Delta\right) y_{i, j}\right)\right|_{x=\xi} \\
& =\left.\left(\sum_{\mu=1}^{m+\vartheta} M_{\vartheta, \mu, \nu} R^{[\mu-1]}\right)\right|_{x=\xi}=\left.\left(\sum_{\mu=1}^{m+\vartheta} M_{\vartheta, \mu, \nu}\left(\sum_{\left(i_{1}, j_{1}\right) \in \Lambda} P_{\left(i_{1}, j_{1}\right)}^{[\mu-1]} y_{i_{1}, j_{1}}\right)\right)\right|_{x=\xi}
\end{aligned}
$$

for any $(i, j) \in \Lambda$. Therefore

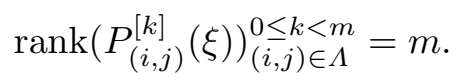

By Lemma 1.7, we have

$$
\begin{aligned}
\vartheta & \leq \operatorname{deg} \Delta-\operatorname{ord}_{x=0} \Delta \\
& \leq m D+\frac{m(m-1)}{2} s-(m-\varepsilon) D+\frac{m(m+1)}{2}+1+\max _{(i, j) \in \Lambda}\left(\operatorname{ord}_{x=0} f_{(i, j)}\right) \\
& =\varepsilon D+\frac{m(m-1)}{2} s+\frac{m(m+1)}{2}+1+\max _{(i, j) \in \Lambda}\left(\operatorname{ord}_{x=0} f_{(i, j)}\right) .
\end{aligned}
$$

Therefore (1.8.1) holds for

$$
\begin{aligned}
D & >\frac{1}{\varepsilon}\left(\frac{m(m-1)}{2} s+\frac{m(m+1)}{2}+1-m+\max _{(i, j) \in \Lambda}\left(\operatorname{ord}_{x=0} f_{(i, j)}\right)\right) \\
& =\frac{1}{\varepsilon}\left(\frac{m(m-1)(s+1)}{2}+1+\max _{(i, j) \in \Lambda}\left(\operatorname{ord}_{x=0} f_{(i, j)}\right)\right) .
\end{aligned}
$$

Notations 1.9. Let $P_{(i, j)}$ be Padé approximants and $R$ be the remainder function in the Padé approximation problem for $f_{(i, j)}$ with parameters $\left(T, D, D_{i}\right)$. Define a sequence $\left\{\bar{p}^{\langle k\rangle}\right\}_{k=0,1, \ldots}$ by

$$
\begin{aligned}
\bar{p}^{\langle k\rangle} & :=\frac{u^{k}}{k !}\left(\frac{d}{d x}+{ }^{t} A\right) \bar{p}^{[0]} \\
& ={ }^{t}\left(P_{(1,1)}^{\langle k\rangle}, \ldots, P_{\left(1, m_{1}\right)}^{\langle k\rangle}, P_{(2,1)}^{\langle k\rangle}, \ldots, P_{\left(n, m_{n}\right)}^{\langle k\rangle}\right) .
\end{aligned}
$$


Furthermore, we write

$$
R^{\langle k\rangle}:=\frac{u^{k}}{k !}\left(\frac{d}{d x}\right)^{k} R
$$

Remark 1.10. Similarly to (1.3.12), one has

$$
R^{\langle k\rangle}={ }^{t} \bar{p}^{\langle k\rangle} \bar{f} \quad(k=0,1,2, \ldots) .
$$

Lemma 1.11. Let $B$ be a differentiable $l \times l^{\prime}$ matrix. Then there exist $q_{k, j} \in \mathbb{Z}[x]$ such that

$$
\left(u\left(\frac{d}{d x}+{ }^{t} A\right)\right)^{k} B=u^{k}\left(\frac{d}{d x}+{ }^{t} A\right)^{k} B+\sum_{j=1}^{k-1} q_{k, j} u^{j}\left(\frac{d}{d x}+{ }^{t} A\right) B
$$

with

$$
\operatorname{deg} q_{k, j} \leq(k-j) s
$$

for $k=1,2, \ldots$ and $j=1, \ldots, k-1$.

Proof. We show (1.11.1) by induction on $k$. For $k=1$, it is trivial. Assume that

$$
\begin{aligned}
& \left(u\left(\frac{d}{d x}+{ }^{t} A\right)\right)^{k} B \\
& \quad=q_{k, k} u^{k}\left(\frac{d}{d x}+{ }^{t} A\right)^{k} B+\sum_{j=1}^{k-1} q_{k, j} u^{j}\left(\frac{d}{d x}+{ }^{t} A\right) B
\end{aligned}
$$

with $q_{k, k}=1$ and $\operatorname{deg} q_{k, j} \leq(k-j) s$ for a given $k \geq 0$ and $j=1, \ldots, k-1$. We now prove it for $k+1$. Since

$$
\begin{aligned}
u\left(\frac{d}{d x}+\right. & \left.{ }^{t} A\right)\left(q_{k, j} u^{j}\left(\frac{d}{d x}+{ }^{t} A\right)^{k} B\right) \\
= & u\left(\frac{d}{d x}\left(q_{k, j} u^{j}\right)+q_{k, j} u^{j} \frac{d}{d x}+q_{k, j} u^{j}{ }^{t} A\right)\left(\frac{d}{d x}+{ }^{t} A\right)^{j} B \\
= & \left(q_{k, j} u^{j+1}\left(\frac{d}{d x}+{ }^{t} A\right)^{j+1}+u \frac{d}{d x}\left(q_{k, j} u^{j}\right)\left(\frac{d}{d x}+{ }^{t} A\right)^{j}\right) B \\
= & \left(q_{k, j} u^{j+1}\left(\frac{d}{d x}+{ }^{t} A\right)^{j+1}+u^{j}\left(\frac{d}{d x} q_{k, j}\right) j q_{k, j}\right. \\
& \left.+\left(\frac{d}{d x} u\right) u^{j}\left(\frac{d}{d x}+{ }^{t} A\right)^{j}\right) B,
\end{aligned}
$$


when we choose

$$
\begin{aligned}
q_{k+1, k+1} & :=1 \\
q_{k+1,1} & :=\frac{d}{d x}\left(q_{k, 1} u\right), \\
q_{k+1, j} & :=\left(\frac{d}{d x} q_{k, j}\right) u+j q_{k, j}\left(\frac{d}{d x} u\right)+q_{k, j-1},
\end{aligned}
$$

we have

$$
\begin{aligned}
& \left(u\left(\frac{d}{d x}+{ }^{t} A\right)\right)^{k+1} B \\
& =u^{k+1} q_{k+1, k+1}\left(\frac{d}{d x}+{ }^{t} A\right)^{k+1} B+\sum_{j=1}^{k} q_{k+1, j} u^{j}\left(\frac{d}{d x}+{ }^{t} A\right)^{j} B .
\end{aligned}
$$

Furthermore, it follows that

$$
\operatorname{deg} q_{k+1,1} \leq \operatorname{deg} q_{k, 1}+\operatorname{deg} u \leq(k-1) s+s=k s,
$$

and

$\operatorname{deg} q_{k+1, j} \leq \max ((k-j) s+s,(k-j+1) s)=(k+1-j) s \quad(j=2, \ldots, k)$.

Lemma 1.12. Let $P_{(i, j)}$ be Padé approximants and $R$ be the remainder function in the Padé approximation problem for $f_{(i, j)}$ with parameters $\left(T, D, D_{i}\right)$. Then $P_{(i, j)}^{\langle k\rangle}$ are Padé approximants and $R^{\langle k\rangle}$ is the remainder function with parameters $\left(T-k, D+k s, D_{i}+k s+k\right)$ for $k=0,1, \ldots, T$.

Proof. It is enough to show that

$$
\begin{gathered}
P_{(i, j)}^{\langle k\rangle} \in \mathbb{Q}[x], \\
\operatorname{deg} P_{(i, j)}^{\langle k\rangle} \leq D+k s, \\
\operatorname{ord}_{x=0} P_{(i, j)}^{\langle k\rangle} \geq D-D_{i}-k, \\
\operatorname{ord}_{x=0} R^{\langle k\rangle} \geq T-k .
\end{gathered}
$$

We show them for any $(i, j)$ by induction on $k$. From Lemma 1.4, the four assertions, (1.12.1)-(1.12.4), are true for $k=0$ and $k=1$. Assume that they are true for a given $k \geq 0$. We now prove them for $k+1$. From Lemma 1.11, we have

$$
\begin{aligned}
(k+1) ! \bar{p}^{\langle k+1\rangle} & =u^{k+1}\left(\frac{d}{d x}+{ }^{t} A\right)^{k} \bar{p}^{\langle 0\rangle} \\
& =\left(u\left(\frac{d}{d x}+{ }^{t} A\right)\right)^{k+1} \bar{p}^{\langle 0\rangle}
\end{aligned}
$$




$$
\begin{aligned}
& -\sum_{h=1}^{k} q_{k+1, h} u^{h}\left(\frac{d}{d x}+{ }^{t} A\right)^{h} \bar{p}^{\langle 0\rangle} \\
= & \bar{p}^{[k+1]}-\sum_{h=1}^{k} q_{k+1, h} h ! \bar{p}^{\langle h\rangle} .
\end{aligned}
$$

Then all entries of $\bar{p}^{\langle k+1\rangle}$ belong to $\mathbb{Q}[x]$. Furthermore, for $h=1, \ldots, k$,

$$
\operatorname{deg}\left(q_{k+1, h} P_{(i, j)}^{\langle h\rangle}\right) \leq(k+1-h) s+D+h s=D+(k+1) s
$$

and

$$
\operatorname{ord}_{x=0}\left(q_{k+1, h} P_{(i, j)}^{\langle h\rangle}\right) \geq D-D_{i}-h \geq D-D_{i}-(k+1) .
$$

From Lemma 1.4, we find

$$
\operatorname{ord}_{x=0} P_{(i, j)}^{[k+1]} \geq D-D_{i}-(k+1) .
$$

Then it follows that

$$
\operatorname{ord}_{x=0} P_{(i, j)}^{\langle k+1\rangle} \geq D-D_{i}-(k+1)
$$

by the identity (1.12.5). The last assertion, $\operatorname{ord}_{x=0} R^{\langle k+1\rangle} \geq T-(k+1)$, is trivial.

Lemma 1.13. Let $P_{(i, j)}$ be Padé approximants and $R$ be the remainder function in the Padé approximation problem for $f_{(i, j)}$ with parameters $\left(T, D, D_{i}\right)$. Suppose that $\bar{f}$ satisfies the differential equation (1.3.3) and that the entries of $\bar{f}$ are linearly independent over $\mathbb{C}(x)$. Then for any number $\xi$ with $\xi u(\xi) \neq 0$ and any $D>\widetilde{D}_{3}$,

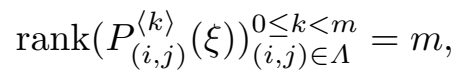

where $\widetilde{D}_{3}$ is a positive constant depending only on $\bar{f}, u, \varepsilon$.

Pr o of. From Lemma 1.11, there exist $q_{k, j} \in \mathbb{Q}(x)$ such that

$$
\bar{p}^{[k]}=k ! \bar{p}^{\langle k\rangle}+\sum_{j=1}^{k-1} j ! q_{k, j} \bar{p}^{\langle j\rangle}
$$

for $k \geq 1$. Putting $L:=[2 \varepsilon D]$, one finds

$$
{ }^{t}\left(\bar{p}^{[0]}, \bar{p}^{[1]}, \ldots, \bar{p}^{[L]}\right)={ }^{t}\left(\bar{p}^{\langle 0\rangle}, \bar{p}^{\langle 1\rangle}, \ldots, \bar{p}^{\langle L\rangle}\right) B
$$

where 


$$
B=\left(\begin{array}{cccccc}
1 & 0 & 0 & 0 & \ldots & 0 \\
0 & 1 & q_{2,1}(x) & q_{3,1}(x) & \ldots & q_{L, 1}(x) \\
0 & 0 & 2 ! & 2 ! q_{3,2}(x) & \ldots & 2 ! q_{L, 2}(x) \\
\vdots & \vdots & \vdots & \ddots & \ldots & \vdots \\
& & & & \ddots & (L-1) ! q_{L, L-1}(x) \\
0 & 0 & \ldots & \ldots & 0 & L !
\end{array}\right) .
$$

Then $\operatorname{det}\left(B_{\mid x=\xi}\right) \neq 0$ for any $\xi \in \mathbb{C}$, and we obtain (1.13.1) from Lemma 1.8 .

\section{Estimations of linear forms}

DeFinitions 2.1. Write

$$
f_{(i, j)}=\sum_{\nu=0}^{\infty} a_{i, j, \nu} x^{\nu} \quad\left(a_{i, j, \nu} \in \mathbb{Q},(i, j) \in \Lambda\right) .
$$

Assume that there exist positive constants $C_{5}$ and $C_{6} \geq 1$, independent of $\nu$, such that

$$
\left|a_{i, j, \nu}\right| \leq C_{5}^{\nu}
$$

and that

$$
\operatorname{den}_{(i, j) \in \Lambda}\left(a_{i, j, 0}, a_{i, j, 1}, \ldots, a_{i, j, \nu}\right) \leq C_{6}^{\nu}
$$

for $\nu=0,1, \ldots$ Then we call $f_{(i, j)} G$-functions; here $\operatorname{den}\left(a_{0}, \ldots, a_{n}\right)$ means the smallest integral positive common denominator of $a_{0}, \ldots, a_{n}$. For convenience's sake, we use a constant $C_{7}$, which is independent of $\nu$, such that

$$
\max _{(i, j) \in \Lambda}\left(\left|C_{6}^{\nu} a_{i, j, 0}\right|,\left|C_{6}^{\nu} a_{i, j, 1}\right|, \ldots,\left|C_{6}^{\nu} a_{i, j, \nu}\right|\right)<C_{7}^{\nu}
$$

for $\nu=0,1, \ldots$

Furthermore, we define the height of $P(x)=a^{(0)}+a^{(1)} x+\ldots+a^{(k)} x^{k} \in$ $M_{\mu_{0} \times \nu_{0}}(\mathbb{Z}[x])$ as

$$
H(P(x)):=\max _{\substack{0 \leq i \leq k \\ 1 \leq \mu \leq \mu_{0} \\ 1 \leq \nu \leq \nu_{0}}}\left|a_{\mu, \nu}^{(i)}\right|,
$$

where $a^{(i)}=\left(a_{\mu, \nu}^{(i)}\right)_{1 \leq \nu \leq \nu_{0}}^{1 \leq \mu \leq \mu_{0}} \in M_{\mu_{0} \times \nu_{0}}(\mathbb{Z})$.

Lemma 2.2. Let $M=\left[\sum_{i=1}^{n} m_{i} D_{i}-\varepsilon D\right]$. There exist $P_{(i, j)} \in \mathbb{Z}[x]$ $((i, j) \in \Lambda)$ such that

$$
\begin{gathered}
\operatorname{deg} P_{(i, j)} \leq D \\
\operatorname{ord}_{x=0} P_{(i, j)} \geq D-D_{i}, \\
H\left(P_{(i, j)}\right) \leq\left(C_{7}^{M} \sum_{i=1}^{n}\left(m_{i} D_{i}+1\right)\right)^{M /(m+\varepsilon D)},
\end{gathered}
$$




$$
\operatorname{ord}_{x=0} \sum_{(i, j) \in \Lambda} P_{(i, j)} f_{(i, j)} \geq M
$$

Proof. Write

$$
P_{(i, j)}=\sum_{\nu=D-D_{i}}^{D} P_{i, j, \nu} x^{\nu}
$$

and

$$
\sum_{(i, j) \in \Lambda} P_{(i, j)} f_{(i, j)}=\sum_{\nu=0}^{\infty} r_{\nu} x^{\nu}
$$

Equating coefficients yields

$$
r_{\nu}=\sum_{(i, j) \in \Lambda} \sum_{k=D-D_{i}}^{\min (\nu, D)} a_{i, j, \nu-k} P_{i, j, k},
$$

for $\nu=0,1, \ldots$ The number of the unknowns $P_{i, j, k}$ in $(2.2 .7)$ is $\sum_{i=1}^{n}\left(m_{i} D_{i}\right.$ $\left.+m_{i}\right)$ and the number of $r_{\nu}$ equal to 0 is $M$. We now consider instead of $(2.2 .7)$,

$$
0=\sum_{(i, j) \in \Lambda} \sum_{k=D-D_{i}}^{\min (\nu, D)}\left(\underset{\substack{\left(i_{1}, j_{1}\right) \in \Lambda \\ 0 \leq h \leq M}}{\operatorname{den}}\left(a_{i_{1}, j_{1}, h}\right)\right) a_{i, j, \nu-k} P_{i, j, k}
$$

with integer coefficients for $\nu=0,1, \ldots, M-1$. Then by Siegel's lemma $[2$, Chapter 2, Lemma 1], there exist non-trivial solutions $P_{i, j, k} \in \mathbb{Z}$ such that

$$
\begin{aligned}
\left|P_{i, j, k}\right| & \leq\left(C_{7}^{M} \sum_{i=1}^{n}\left(m_{i} D_{i}+1\right)\right)^{M /\left(\sum_{i=1}^{n}\left(m_{i} D_{i}+m_{i}\right)-M\right)} \\
& \leq\left(C_{7}^{M} \sum_{i=1}^{n}\left(m_{i} D_{i}+1\right)\right)^{M /(m+\varepsilon D)}
\end{aligned}
$$

Definitions 2.3. For $A$ being the coefficient of the differential equation (1.3.3), we define a sequence $\left\{A_{k}\right\}_{k=0,1, \ldots} \subset M_{m}(\mathbb{Q}(x))$ by induction as

$$
\begin{aligned}
A_{0} & =I \quad(\text { the identity matrix }), \\
A_{k+1} & =\frac{d}{d x} A_{k}+A_{k} A \quad(k=1,2, \ldots) .
\end{aligned}
$$

Furthermore, we write

$$
\delta_{\nu}:=\operatorname{den}_{0 \leq k \leq \nu}\left(\text { the coefficients of the entries of } \frac{u^{k}}{k !} A_{k}\right)
$$


for $\nu=0,1, \ldots$ We say that $A \in M_{m}(\mathbb{Q}(x))$ has the $(G, C)$-property when there exists a constant $\delta>0$, independent of $\nu$, such that

$$
\delta_{\nu} \leq \delta^{\nu} .
$$

Remark 2.4. One finds immediately that

$$
\frac{u^{k}}{k !} A_{k} \in M_{m}(\mathbb{Q}[x]) .
$$

The following lemma is due to D. V. Chudnovsky and G. V. Chudnovsky. Their method is the Padé approximations of the second kind (cf. [4]).

Lemma 2.5 (D. V. Chudnovsky, G. V. Chudnovsky, cf. [4]). If there exists a solution of the differential equation (1.3.3) such that its entries are $G$-functions and they are linearly independent over $\mathbb{Q}(x)$, then $A$ has the $(G, C)$-property.

Proof. We find the proof in [4, Theorem III].

Lemma 2.6. Let $P \in M_{m}(\mathbb{Z}[x])$. For $k=0,1, \ldots$,

$$
\frac{\delta_{k}}{k !} u^{k}\left(\frac{d}{d x}+{ }^{t} A\right)^{k} P \in M_{m}(\mathbb{Z}[x]) .
$$

Pr o o f. We assume that $\bar{y}$ is a solution of the differential equation (1.3.3). Applying $d / d x$ to $\bar{y} k$ times gives

$$
\left(\frac{d}{d x}\right)^{k} \bar{y}=A_{k} \bar{y}
$$

Write $R={ }^{t} P \bar{y}$. Arguing as for the identities (1.3.12) yields

$$
\left(\frac{d}{d x}\right)^{k} R={ }^{t}\left(\left(\frac{d}{d x}+{ }^{t} A\right)^{k} P\right) \bar{y} .
$$

Now one has

$$
\begin{aligned}
\frac{1}{k !}\left(\frac{d}{d x}\right)^{k} R & =\frac{1}{k !} \sum_{h=0}^{k}\left(\begin{array}{l}
k \\
h
\end{array}\right)\left(\left(\frac{d}{d x}\right)^{k-h}{ }^{t} P\right)\left(\frac{d}{d x}\right)^{h} \bar{y} \\
& =\sum_{h=0}^{k}\left(\left(\frac{d}{d x}\right)^{k-h}{ }^{t} P\right) \frac{1}{k !} A_{h} \bar{y} .
\end{aligned}
$$

Then

$$
\frac{\delta_{k}}{k !} u^{k}\left(\left(\frac{d}{d x}+{ }^{t} A\right)^{k} P\right) \bar{y}=\sum_{h=0}^{k}\left(\frac{u^{k-h}}{(k-h) !} u^{k-h}{ }^{t} P\right) \frac{\delta_{k}}{h !} u^{h} A_{h} \bar{y} .
$$


Since

$$
\frac{1}{(k-h) !}\left(\frac{d}{d x}\right)^{k-h}{ }^{t} P \in M_{m}(\mathbb{Z}[x]) \quad \text { and } \quad \frac{\delta_{k}}{h !} u^{h} A_{h} \in M_{m}(\mathbb{Z}[x])
$$

for $h \leq k$, we get (2.6.1).

From now on, we consider the Padé approximations of Lemma 2.2.

Lemma 2.7. Let $P_{(i, j)}$ be the Padé approximants obtained in Lemma 2.2. Suppose that $A$ in (1.3.3) has the $(G, C)$-property. For any $M_{0} \leq[2 \varepsilon D]$,

$$
\begin{gathered}
\operatorname{deg} P_{(i, j)}^{\langle k\rangle} \leq D-k s, \\
\operatorname{ord}_{x=0} P_{(i, j)}^{\langle k\rangle} \geq D-D_{i}-k, \\
\delta_{M_{0}} P_{(i, j)}^{\langle k\rangle} \in \mathbb{Z}[x], \\
H\left(\delta_{M_{0}} P_{(i, j)}^{\langle k\rangle} \leq \leq\left(C_{7}^{M} \sum_{i=1}^{n}\left(m_{i} D_{i}+1\right)\right)^{M /(m+\varepsilon D)} \delta^{M_{0}}\left(m(s+1)^{2} H_{0}\right)^{k}\right. \\
\times \frac{1}{k !} \prod_{h=1}^{k}(D+(h-1)(s+1)+2),
\end{gathered}
$$

where $M=\left[\sum_{i=1}^{n} m_{i} D_{i}-\varepsilon D\right]$ and $H_{0}=\max (H(u), H(u A))$. Furthermore, for $\xi \in \mathbb{R}$ with $|\xi|<C_{5}^{-1}$,

$$
\left|\delta_{M_{0}} R_{\mid x=\xi}^{\langle k\rangle}\right| \leq m(D+k s) \max _{(i, j) \in \Lambda}\left(H\left(\delta_{M_{0}} P_{(i, j)}^{\langle k\rangle}\right)\right) \frac{\left|C_{5} \xi\right|^{M-k}}{1-\left|C_{5} \xi\right|}
$$

for any $k \leq M_{0}$.

P r o o f. (2.7.1) and (2.7.2) hold by Lemma 1.12. (2.7.3) holds by Lemma 2.6. We show (2.7.4) by induction on $k$. It is true for $k=0$ by Lemma 2.2. Assume that it is true for a given $k \geq 0$. For $k+1$, we have

$$
\begin{aligned}
& u\left(\frac{d}{d x}+{ }^{t} A\right)\left(u^{k}\left(\frac{d}{d x}+{ }^{t} A\right)^{k}\right) \\
& =u \frac{d}{d x}\left(u^{k}\left(\frac{d}{d x}+{ }^{t} A\right)^{k}\right)+u^{k+1}{ }^{t} A\left(\frac{d}{d x}+{ }^{t} A\right)^{k} \\
& =k\left(\frac{d}{d x} u\right) u^{k}\left(\frac{d}{d x}+{ }^{t} A\right)^{k}+u^{k+1} \frac{d}{d x}\left(\frac{d}{d x}+{ }^{t} A\right)^{k}+u^{k+1}{ }^{t} A\left(\frac{d}{d x}+{ }^{t} A\right)^{k} \\
& =u^{k+1}\left(\frac{d}{d x}+{ }^{t} A\right)^{k+1}+k\left(\frac{d}{d x} u\right) u^{k}\left(\frac{d}{d x}+{ }^{t} A\right)^{k},
\end{aligned}
$$

or 


$$
\begin{aligned}
& u^{k+1}\left(\frac{d}{d x}+{ }^{t} A\right)^{k+1} \\
& =u\left(\frac{d}{d x}+{ }^{t} A\right)\left(u\left(\frac{d}{d x}+{ }^{t} A\right)^{k}\right)-k\left(\frac{d}{d x} u\right) u^{k}\left(\frac{d}{d x}+{ }^{t} A\right)^{k} .
\end{aligned}
$$

It yields

$$
\delta_{M_{0}}(k+1) ! \bar{p}^{\langle k+1\rangle}=\delta_{M}\left(u\left(\frac{d}{d x}+{ }^{t} A\right)-k\left(\frac{d}{d x} u\right)\right) k ! \bar{p}^{\langle k\rangle} .
$$

Since $\operatorname{deg} P_{(i, j)}^{\langle k\rangle} \leq D+k s$ and $\operatorname{deg} u \leq s+1$, the height of every component of the right side of (2.7.7) is not greater than

$$
\begin{aligned}
k !\left((s+2)(D+k s+1) H_{0}+m(s+1) H_{0}\right. & \left.+k(s+2)^{2} H_{0}\right) \\
& \times \max _{(i, j) \in \Lambda} H\left(\delta_{M_{0}} P_{(i, j)}^{\langle k\rangle}\right) \\
\leq k ! m H_{0}(s+2)^{2}(D+k(s+1)+2) & \max _{(i, j) \in \Lambda} H\left(\delta_{M_{0}} P_{(i, j)}^{\langle k\rangle}\right) .
\end{aligned}
$$

Therefore we obtain

$$
\begin{aligned}
H\left(\delta_{M_{0}} P_{(i, j)}^{\langle k+1\rangle}\right) \leq\left(C_{7}^{M} \sum_{i=1}^{n}\left(m_{i} D_{i}+1\right)\right)^{M /(m+\varepsilon D)} \delta^{M_{0}}\left(m(s+2)^{2} H_{0}\right)^{k+1} \\
\times \frac{1}{(k+1) !} \prod_{h=1}^{k+1}(D+(h-1)(s+1)+2) .
\end{aligned}
$$

So (2.7.4) holds for any $k \leq M_{0}$.

Now since $\operatorname{ord}_{x=0} R^{\langle k\rangle} \geq M-k$ and $\operatorname{deg} P_{(i, j)}^{\langle k\rangle} \leq D+j s$, one can write

$$
\delta_{M_{0}} R^{\langle k\rangle}(\xi)=\sum_{(i, j) \in \Lambda} \delta_{M_{0}} P_{(i, j)}^{\langle k\rangle}(\xi) f_{(i, j)}(\xi)=\sum_{\nu=M-k}^{\infty} r_{\nu}^{\langle k\rangle} x^{\nu} \in \mathbb{Q}[[x]]
$$

It follows that

$$
\left|r_{\nu}^{\langle k\rangle}\right| \leq m(D+k s+1) \max _{(i, j) \in \Lambda} H\left(\delta_{M_{0}} P_{(i, j)}^{\langle k\rangle}\right) C_{5}^{\nu} .
$$

Hence we obtain the inequality (2.7.5).

Lemma 2.8. Let $a, b \in \mathbb{Z} \backslash\{0\}$ with $|a / b| \leq 1$ and let $p(x) \in \mathbb{Z}[x]$ with $\operatorname{deg} p(x) \leq N$ and $\operatorname{ord}_{x=0} p(x) \geq N-T$. Then $p(a / b) b^{N} \in \mathbb{Z}$ and

$$
\left|p\left(\frac{a}{b}\right) b^{N}\right| \leq(T+1) H(p(x))|a|^{N-T}|b|^{T} .
$$

Proof. Let $p(x)=c_{N-T} x^{N-T}+\ldots+c_{N} x^{N} \in \mathbb{Z}[x]$. Then

$$
p\left(\frac{a}{b}\right) b^{N}=a^{N-T}\left(c_{N-T} b^{T}+\ldots+c_{N} a^{T}\right) \in \mathbb{Z}
$$


and

$$
\begin{aligned}
\left|p\left(\frac{a}{b}\right) b^{N}\right| & \leq|a|^{N-T}(T+1)|b|^{T} \max _{N-T \leq k \leq N}\left|c_{k}\right| \\
& =(T+1) H(p(x))|a|^{N-T}|b|^{T} .
\end{aligned}
$$

Lemma 2.9. Let $M=\left[\sum_{i=1}^{n} m_{i} D_{i}-\varepsilon D\right]$ and $M_{0}=[2 \varepsilon D]$. Then for any $\xi \in \mathbb{R}$ with $\left|C_{5} \xi\right| \leq \gamma_{0}$ with a positive constant $\gamma_{0}<1$ there exists a positive constant $\gamma$, independent of $D$ and $\xi$, such that

$$
\gamma^{D} \geq m\left(D+M_{0} s+1\right) \max _{(i, j) \in \Lambda}\left(H\left(\delta_{M_{0}} P_{(i, j)}^{\left\langle M_{0}\right\rangle}\right)\right) \frac{\left|C_{5}\right|^{M}}{1-\left|C_{5} \xi\right|}
$$

for any $D \geq \widetilde{D}_{4}$; here $\widetilde{D}_{4}$ is a positive constant depending only on $m, \varepsilon, u$ and $\gamma_{0}$.

Pr o of. From Lemma 2.7, in order to prove Lemma 2.9, it suffices to show the existence of a constant $\gamma$, independent of $\xi$ and any sufficiently large $D$, such that

$$
\begin{aligned}
\gamma^{D} \geq & \delta_{M_{0}}\left(C_{7}^{M} \sum_{i=1}^{n}\left(m_{i} D_{i}+1\right)\right)^{M /(m+\varepsilon D)} \\
& \times\left(m(s+2)^{2} H_{0}\right)^{M_{0}} m\left(D+M_{0} s+1\right) \\
& \times \frac{1}{M_{0} !} \prod_{h=1}^{M_{0}}(D+(h-1)(s+1)+2) \frac{\left|C_{5}\right|^{M}}{1-\gamma_{0}} .
\end{aligned}
$$

Now for any sufficiently large $D$, one has

$$
\begin{gathered}
\frac{M}{m+\varepsilon D} \leq \frac{m}{\varepsilon}, \\
m\left(D+M_{0} s+1\right) \leq m((1+2 \varepsilon s) D+1) \leq e^{D}
\end{gathered}
$$

and there exists a positive constant $\gamma_{1}$, independent of $D$ and depending only on $\varepsilon$ and $s$, such that

$$
\begin{aligned}
& \frac{1}{M_{0} !} \prod_{h=1}^{M_{0}}(D+(h-1)(s+1)+2) \\
& \leq \frac{1}{M_{0} !}\left(D+M_{0}(s+1)+1\right)^{M_{0}} \leq \frac{(D+2 \varepsilon D(s+1)+1)^{M_{0}}}{M_{0} !} \\
& \underset{D \rightarrow \infty}{\approx} \frac{((1+2 \varepsilon(s+1)) D+1)^{M_{0}}}{\sqrt{2 \pi M_{0}} M_{0}^{M_{0}} e^{-M_{0}}} \leq e^{M_{0}}\left(\frac{2+2 \varepsilon(d+1)}{2 \varepsilon}\right)^{M_{0}} \leq \gamma_{1}^{D} .
\end{aligned}
$$

This gives the existence of $\gamma$ independent of $D$ and $\xi$ and depending only on $\delta, \varepsilon, C_{7}, m, s, H_{0}, m, s, C_{5}, \gamma_{0}$, such that the right side of (2.9.2) does 
not exceed

$$
\delta^{2 \varepsilon D} C_{7}^{n^{2} D / \varepsilon}\left(n(s+2)^{2} H_{0}\right)^{2 \varepsilon D} e^{D} \gamma_{1}^{D}\left|C_{5}\right|^{n D} \frac{1}{1-\gamma_{0}} \leq \gamma^{D}
$$

for $D>\widetilde{D}_{4}$. We choose $\widetilde{D}_{4}$ to be a positive constant depending only on $m$, $\varepsilon, s, \gamma_{0}$.

LEMMA 2.10. Under the assumptions of Lemma 2.9, for any $\xi=a / b$ with $a, b \in \mathbb{Z}$ and $\xi u(\xi) \neq 0$, there exist $P_{(i, j)}^{(k, l)} \in \mathbb{Z}((i, j) \in \Lambda)$ such that

$$
\begin{gathered}
\operatorname{det}\left(P_{(i, j)}^{(k, l)}\right)_{(i, j),(k, l) \in \Lambda} \neq 0, \\
\left|P_{(i, j)}^{(k, l)}\right| \leq\left(D_{k}+(s+1) M_{0}+1\right) \\
\times \max _{\left(i_{1}, j_{1}\right) \in \Lambda} H\left(\delta_{M_{0}} P_{\left(i_{1}, j_{1}\right)}^{\left\langle M_{0}\right\rangle}\right)|a|^{D-D_{k}}|b|^{D_{k}+(s+1) M_{0}}, \\
\left|\sum_{(k, l) \in \Lambda} P_{(i, j)}^{(k, l)} f_{(k, l)}(\xi)\right| \leq \gamma^{D}|a|^{M-M_{0}}|b|^{-M+D+(s+1) M_{0}}
\end{gathered}
$$

for $D>\widetilde{D}_{5}$; here $\widetilde{D}_{5}$ is a positive constant depending only on $\bar{f}, s, \varepsilon$, $m, \gamma_{0}$.

Proof. According to Lemma 1.13, let $\lambda_{i, j} \in \mathbb{N} \cup\{0\}((i, j) \in \Lambda)$ be distinct indices with $\max _{(i, j) \in \Lambda} \lambda_{i, j} \leq M_{0}$ such that

$$
\operatorname{rank}\left(P_{(k, l)}^{\left\langle\lambda_{i, j}\right\rangle}(\xi)\right)_{(i, j),(k, l) \in \Lambda}=m
$$

Now we write

$$
P_{(i, j)}^{(k, l)}=\delta_{M_{0}} P_{(k, l)}^{\left\langle\lambda_{i, j}\right\rangle}(\xi) b^{D+\lambda_{i, j} s} .
$$

Then $\operatorname{det}\left(P_{(i, j)}^{(k, l)}\right)_{(i, j),(k, l) \in \Lambda} \neq 0$. Since $\operatorname{deg} P_{(k, l)}^{\left\langle\lambda_{i, j}\right\rangle} \leq D+\lambda_{i, j} s$ and $\operatorname{ord}_{x=0} P_{(k, l)}^{\left\langle\lambda_{i, j}\right\rangle} \geq D-D_{k}-\lambda_{i, j}$ by Lemma 1.13, we have

$$
\begin{aligned}
\left|P_{(i, j)}^{(k, l)}\right| \leq & \left(D_{k}+\lambda_{i, j}(s+1)+1\right) \\
& \times \max _{\left(i_{1}, j_{1}\right) \in \Lambda} H\left(\delta_{M_{0}} P_{\left(i_{1}, j_{1}\right)}^{\left\langle\lambda_{i, j}\right\rangle}\right)|a|^{D-D_{k}-\lambda_{i, j}}|b|^{D_{k}+\lambda_{i, j}(s+1)} \\
\leq & \left(D_{k}+M_{0}(s+1)\right) \\
& \times \max _{\left(i_{1}, j_{1}\right) \in \Lambda}\left(H\left(\delta_{M_{0}} P_{(i, j)}^{\left\langle M_{0}\right\rangle}\right)\right)|a|^{D-D_{k}}|b|^{D_{k}+M_{0}(s+1)} .
\end{aligned}
$$

From Lemmas 2.7 and 2.9, one finds 


$$
\begin{aligned}
& \left|\sum_{(k, l) \in \Lambda} \delta_{M_{0}} P_{(k, l)}^{\left\langle\lambda_{i, j}\right\rangle}(\xi) f_{(k, l)}(\xi)\right| \\
& \quad \leq n\left(D+\lambda_{i, j} s+1\right) \max _{\left(k_{1}, l_{1}\right) \in \Lambda}\left(H\left(\delta_{M_{0}} P_{\left(k_{1}, l_{1}\right)}^{\left\langle\lambda_{i, j}\right\rangle}\right)\right) \frac{\left|C_{5} \xi\right|^{M-\lambda_{i, j}}}{1-\left|C_{5} \xi\right|} \\
& \quad \leq n\left(D+M_{0} s+1\right) \max _{\left(k_{1}, l_{1}\right) \in \Lambda}\left(H\left(\delta_{M_{0}} P_{\left(k_{1}, l_{1}\right)}^{\left\langle M_{0}\right\rangle}\right)\right) \frac{C_{5}^{M}}{1-\left|C_{5} \xi\right|} \\
& \quad \leq \gamma^{D}|\xi|^{M-\lambda_{i, j}}
\end{aligned}
$$

for any $\xi$ with $|\xi| \leq \gamma_{0} C_{5}^{-1} \leq 1$. Therefore we obtain

$$
\begin{aligned}
\left|\sum_{(k, l) \in \Lambda} P_{(i, j)}^{(k, l)} f_{(k, l)}(\xi)\right| & \leq \gamma^{D}|\xi|^{M-\lambda_{i, j}}|b|^{D+\lambda_{i, j} s} \\
& =\gamma^{D}|a|^{M-\lambda_{i, j}}|b|^{-M+D+(s+1) \lambda_{i, j}} \\
& \leq \gamma^{D}|a|^{M-M_{0}}|b|^{-M+D+(s+1) M_{0}}
\end{aligned}
$$

3. Proof of Theorem $\mathbf{0 . 2}$. We now prove the following theorem instead of Theorem 0.2 .

Theorem 3.1. Let $A_{(i)} \in M_{m_{i}}(\mathbb{Q}(x)), m=\sum_{i=1}^{n} m_{i}$ and let $\Lambda:=$ $\left\{(i, j) \mid i=1, \ldots, n ; j=1, \ldots, m_{i}\right\}$. Suppose that $f_{(i, j)} \in \mathbb{Q}[[x]]((i, j) \in \Lambda)$ are $G$-functions which satisfy

$$
\frac{d}{d x}\left(\begin{array}{c}
f_{(1,1)} \\
\vdots \\
f_{\left(1, m_{1}\right)} \\
f_{(2,1)} \\
\vdots \\
f_{\left(n, m_{n}\right)}
\end{array}\right)=A\left(\begin{array}{c}
f_{(1,1)} \\
\vdots \\
f_{\left(1, m_{1}\right)} \\
f_{(2,1)} \\
\vdots \\
f_{\left(n, m_{n}\right)}
\end{array}\right)
$$

where

$$
A=\left(\begin{array}{ccc}
A_{(1)} & & 0 \\
& \ddots & \\
0 & & A_{(n)}
\end{array}\right) .
$$

Furthermore, assume that $f_{(1,1)}, \ldots, f_{\left(n, m_{n}\right)}$ are linearly independent over $\mathbb{Q}(x)$. Let $r=a / b \in \mathbb{Q}, a, b \in \mathbb{Z}, r \neq 0$, and $0<\varepsilon_{0}<1 / 2$ be a fixed real number. Then there exist an effective constant $C_{8}$, depending only on $f_{(i, j)}, \varepsilon_{0}, m, A$, and an effective constant $C_{9}$, depending only on $f_{(i, j)}, \varepsilon, m$, $r, A$, such that if

$$
|b|^{\varepsilon_{0}}>C_{8}|a|^{2 m(m+1)},
$$


then for any $H_{i, j} \in \mathbb{Z}$ such that $H:=\max _{(i, j) \in \Lambda}\left|H_{i, j}\right|>C_{9}$, and for $H_{i}:=\max _{1 \leq j \leq m_{i}}\left(\left|H_{i, j}\right|, 1\right)$,

$$
\left|\sum_{(i, j) \in \Lambda} H_{i, j} f_{(i, j)}(r)\right|>\frac{H^{1-\varepsilon_{0}}}{H_{1}^{m_{1}} \cdots H_{n}^{m_{n}}} .
$$

Proof. Here is a sketch of the proof: Using the estimates of the first sections, we obtain the inequalities (3.1.23), which involve the data and the parameters $D, D_{1}, \ldots, D_{n}$. These inequalities yield the bound in Theorem 3.1 provided that $D, D_{1}, \ldots, D_{n}$ satisfy the two conditions $(3.1 .25)$ and (3.1.26). The condition (3.1.25) will transform into (3.1.27). Then we prove the existence of Padé polynomials with these conditions for any $0<\varepsilon<1 / 2$ and for any large $H$.

After renumbering the indices $(k, l)$ of $P_{(i, j)}^{(k, l)}$ obtained in Lemma 2.10, we can assume that

$$
V:=\left(\begin{array}{ccc}
H_{1,1} & \ldots & H_{n, m_{n}} \\
P_{(1,2)}^{(1,1)} & \ldots & P_{(1,2)}^{\left(n, m_{n}\right)} \\
\vdots & & \vdots \\
P_{\left(n, m_{n}\right)}^{(1,1)} & \ldots & P_{\left(n, m_{n}\right)}^{\left(n, m_{n}\right)}
\end{array}\right)
$$

is non-singular. Let $\Delta:=\operatorname{det} V$ and $\lambda_{i, j}:=\sum_{\mu=1}^{i-1} m_{\mu}+j$ and let $\Delta_{(i, j)}^{(k, l)}$ denote the $\left(\lambda_{i, j}, \lambda_{k, l}\right)$ th cofactor of $V$. Without loss of generality, we put $H:=\left|H_{1,1}\right|=\max _{(i, j) \in \Lambda}\left|H_{i, j}\right|$ and assume that $f_{(1,1)}(r) \neq 0$. We write

$L_{(1,1)}:=\sum_{(k, l) \in \Lambda} H_{k, l} f_{(k, l)}(r), \quad L_{(i, j)}:=\sum_{(k, l) \in \Lambda} P_{(i, j)}^{(k, l)} f_{(k, l)}(r) \quad\left((i, j) \in \Lambda^{\prime}\right)$,

where

$$
\Lambda^{\prime}:=\Lambda \backslash\{(1,1)\}
$$

It follows that

$$
f_{(1,1)} \Delta=L_{(1,1)} \Delta_{(1,1)}^{(1,1)}+\sum_{(i, j) \in \Lambda^{\prime}} L_{(i, j)} \Delta_{(i, j)}^{(1,1)}
$$

Put

$$
M:=\left[\sum_{i=1}^{n} m_{i} D_{i}-\varepsilon D\right] \quad \text { and } \quad M_{0}:=[2 \varepsilon D] .
$$

Arguing as in Lemmas 2.9 and 2.10, one finds that there exists a positive constant $\widetilde{\gamma}$, independent of $r, D, D_{i}$, such that

$$
\begin{aligned}
\left|P_{(i, j)}^{(k, l)}\right| \leq & \left(D_{k}+M_{0}(s+1)+1\right) \\
& \times \max _{\left(i_{1}, j_{1}\right) \in \Lambda}\left(H\left(\delta_{M_{0}} P_{\left(i_{1}, j_{1}\right)}^{\left\langle M_{0}\right\rangle}\right)\right)|a|^{D-D_{k}}|b|^{D_{k}+M_{0}(s+1)} \\
\leq & \widetilde{\gamma}^{D / m}|a|^{D-D_{k}}|b|^{D_{k}+(s+1) M_{0}}
\end{aligned}
$$


for $D>\widetilde{D}_{6}$; here $\widetilde{D}_{6}$ depends only on $m, \varepsilon, u, \gamma_{0}$. Then we have

$$
\left|\Delta_{(1,1)}^{(1,1)}\right| \leq(m-1) ! \widetilde{\gamma}^{D} \prod_{(\mu, \nu) \in \Lambda^{\prime}}|a|^{D-D_{\mu}}|b|^{D_{\mu}+(s+1) M_{0}}
$$

and

$$
\begin{aligned}
& \left|\Delta_{(i, j)}^{(1,1)}\right| \\
\leq & (m-2) ! \widetilde{\gamma}^{D} \sum_{(\mu, \nu) \in \Lambda^{\prime}} \frac{|a|^{\Sigma_{(k, l) \in \Lambda^{\prime}} D-D_{k}}|b|^{\Sigma_{(k, l) \in \Lambda^{\prime}} D_{k}+(m-1)(s+1) M_{0}}}{|a|^{D-D_{\mu}}|b|^{D_{\mu}+(s+1) M_{0}}} H_{\mu} .
\end{aligned}
$$

The inequality (2.10.3) in Lemma 2.10 gives

$$
\left|L_{(i, j)}\right| \leq \gamma^{D}|a|^{M-M_{0}}|b|^{-M+D+(s+1) M_{0}} \quad\left((i, j) \in \Lambda^{\prime}\right) .
$$

Thus by (3.1.6), we have

$$
\text { (3.1.11) } \begin{aligned}
\left|f_{(1,1)}(r) \Delta\right| \leq & (m-1) !(\gamma \widetilde{\gamma})^{D}|a|^{M-M_{0}+\Sigma_{(k, j) \in \Lambda^{\prime}}\left(D-D_{k}\right)} \\
& \times|b|^{-M+\Sigma_{(k, j) \in \Lambda^{\prime}} D_{k, l}+m(s+1) M_{0}} \\
& \times \sum_{(k, l) \in \Lambda^{\prime}} \frac{H_{\mu}}{|a|^{D-D_{\mu}}|b|^{D_{\mu}+(s+1) M_{0}}} \\
& +\left|L_{(1,1)}\right|(m-1) ! \widetilde{\gamma}^{D} \prod_{(\mu, \nu) \in \Lambda^{\prime}}|a|^{D-D_{\mu}}|b|^{D_{\mu}+(s+1) M_{0}}
\end{aligned}
$$

Now we put

$$
\varepsilon_{1}:=\frac{\sum_{i=1}^{n} m_{i} D_{i}-M}{D} .
$$

This $\varepsilon_{1}$ satisfies $\varepsilon \leq \varepsilon_{1} \leq \varepsilon+1 / D<\frac{3}{2} \varepsilon$ for any sufficiently large $D$. We choose the parameters

$$
D_{1}:=D
$$

and $D_{2}, D_{3}, \ldots, D_{n}$ to be the smallest integers satisfying

$$
\begin{aligned}
\left|f_{(1,1)}(r)\right|^{-1} H_{i} m !(\gamma \widetilde{\gamma})^{D}|a|^{M-M_{0}+\Sigma_{(k, l) \in \Lambda^{\prime}}\left(D-D_{k}\right)} & \\
& \times|b|^{-M+\Sigma_{(k, l) \in \Lambda^{\prime}} D_{k}+m(s+1) M_{0}} \\
(= & \left.\left|f_{(1,1)}(r)\right|^{-1} H_{i} m !(\gamma \widetilde{\gamma})^{D}|a|^{m D-\varepsilon_{1} D-M_{0}}|b|^{\varepsilon_{1} D+m(s+1) M_{0}}\right) \\
\leq & |a|^{D-D_{i}}|b|^{D_{i}+(s+1) M_{0}} .
\end{aligned}
$$

We need, for $i=1, \ldots, n$,

$$
D \geq D_{i}
$$

and

$$
D_{i} \geq 2 \varepsilon D
$$


in Definitions 1.1. First, we show (3.1.16). Consider the following inequality which is equivalent to (3.1.14):

$$
\begin{aligned}
H_{i} \leq & \frac{\left|f_{(1,1)}\right|}{m !}(\gamma \widetilde{\gamma})^{D}|a|^{-D_{i}-(m-1) D+\varepsilon_{1} D+M_{0}} \\
& \times|b|^{D_{i}-\varepsilon_{1} D-(m-1)(s+1) M_{0}} .
\end{aligned}
$$

We define a function $g$ of $D$ by

$$
g:=\frac{\log (m !)-\log \left|f_{(1,1)}(r)\right|}{D \log |b|}+\frac{\log (\gamma \widetilde{\gamma})}{\log |b|},
$$

or

$$
|b|^{g}=\left(\sqrt[D]{\frac{m !}{\left|f_{(1,1)}(r)\right|}}\right)(\gamma \widetilde{\gamma})
$$

Now we choose the constant $C_{8}$ in Theorem 3.1 to satisfy

$$
C_{8} \geq(\gamma \widetilde{\gamma})^{3 \varepsilon_{0} / \varepsilon}
$$

From the assumption

$$
|b|^{\varepsilon_{0}} \geq C_{8}
$$

one has $|b|^{\varepsilon} \geq(\gamma \widetilde{\gamma})^{3}$ and $0 \leq g<\varepsilon / 2$ for any sufficiently large $D$. (The lower bound of $D$ depends on $m$ and $f_{(1,1)}(r)$.) Moreover, we put

$$
\eta:=\frac{\log |a|}{\log |b|} .
$$

Then $0<\eta \leq 1$. With these notations, (3.1.17) is equivalent to

(3.1.21) $\log H_{i} \leq\left(-g D-\eta D_{i}-(m-1) \eta D+\varepsilon_{1} \eta D\right.$

$$
\begin{aligned}
& \left.+\eta M_{0}+D_{i}-\varepsilon D-(m-1)(s+1) M_{0}\right) \log |b| \\
& =\left(D_{i}(1-\eta)-D\left(g+\left(m-1-\varepsilon_{1}\right) \eta+\varepsilon_{1}\right.\right. \\
& \left.\left.+((m-1)(s+1)-\eta) \frac{M_{0}}{D}\right)\right) \log |b| .
\end{aligned}
$$

Since $H_{i} \geq 1$, the coefficient on $\log |b|$ on the right side of (3.1.21) (= the exponent of $|b|$ ) is non-negative, that is to say,

$$
D_{i} \geq \frac{g+\left(m-1-\varepsilon_{1}\right) \eta+\varepsilon_{1}+((m-1)(s+1)-\eta) M_{0} / D}{1-\eta} .
$$

Thus (3.1.16) holds for $i=1, \ldots, n$ since $\varepsilon_{1}>\varepsilon$ and $M_{0} / D \geq 2 \varepsilon-1 / D$ for any sufficiently large $D$. 
Now applying (3.1.14) in (3.1.11) yields

(3.1.23)

$$
\begin{aligned}
& \left|f_{(1,1)}(r) \Delta\right| \\
& \leq(m-1) ! \sum_{(\mu, \nu) \in \Lambda^{\prime}} \frac{\left|f_{(1,1)}(r)\right|}{m !}+\left|L_{(1,1)}\right|(m-1) ! \widetilde{\gamma}^{D} \prod_{(\mu, \nu) \in \Lambda^{\prime}} H_{\mu} \\
& \quad \times \prod_{(\mu, \nu) \in \Lambda^{\prime}}\left(\frac{m !}{\left|f_{(1,1)}(r)\right|}(\gamma \widetilde{\gamma})^{D}|a|^{M-M_{0}+\Sigma_{(k, l) \in \Lambda^{\prime}}\left(D-D_{k}\right)-1}\right. \\
& \left.\quad \times|b|^{-M+D+\Sigma_{(k, l) \in \Lambda^{\prime}} D_{k}+1+m(s+1) M_{0}}\right) \\
& =\frac{m-1}{m}\left|f_{(1,1)}\right|+\left|L_{(1,1)}\right|(m-1) ! \widetilde{\gamma}^{D}\left(\prod_{(\mu, \nu) \in \Lambda^{\prime}} H_{\mu}\right) \\
& \quad \times \prod_{(\mu, \nu) \in \Lambda^{\prime}} \frac{m !}{\left|f_{(1,1)}(r)\right|}(\gamma \widetilde{\gamma})^{D}|a|^{m D-\varepsilon_{0} D-M_{0}-1}|b|^{\varepsilon_{1} D+m(s+1) M_{0}+1} .
\end{aligned}
$$

Write

$$
\begin{aligned}
\mathcal{E}:=( & m-1) ! \widetilde{\gamma}^{D} \\
& \times\left(\frac{m !}{\left|f_{(1,1)}(r)\right|}(\gamma \widetilde{\gamma})^{D}|a|^{m D-\varepsilon_{1} D-M_{0}-1}|b|^{\varepsilon_{1} D+m(s+1) M_{0}+1}\right)^{m-1} .
\end{aligned}
$$

Since

$$
\Delta \in \mathbb{Z} \backslash\{0\}
$$

and by (3.1.23), we have

$$
\left|f_{(1,1)}(r)\right| \leq \frac{m-1}{m}\left|f_{(1,1)}(r)\right|+\left|L_{(1,1)}\right| \mathcal{E} \prod_{(\mu, \nu) \in \Lambda^{\prime}} H_{\mu},
$$

or

$$
\begin{aligned}
\left|L_{(1,1)}\right| & \geq \frac{\left|f_{(1,1)}(r)\right|}{m} \mathcal{E}^{-1} \prod_{(\mu, \nu) \in \Lambda^{\prime}} H_{\mu} \\
& =\frac{\left|f_{(1,1)}(r)\right|}{m} \mathcal{E}^{-1} \frac{H}{H_{1}^{m_{1}} \cdots H_{n}^{m_{n}}} .
\end{aligned}
$$

Consequently, the bound (3.1.3) requires

(3.1.25) the smallest integer $D_{i}$ in (3.1.14) (or (3.1.21)) satisfies (3.1.15) and

or more weakly

$$
H^{-\varepsilon_{0}}<\frac{\left|f_{(1,1)}(r)\right|}{m} \mathcal{E}^{-1}
$$




$$
\begin{aligned}
H^{\varepsilon_{0}}> & \left(\frac{m !}{\left|f_{(1,1)}(r)\right|}(\gamma \widetilde{\gamma})^{D}\right)^{m} \\
& \times\left(|a|^{m D-\varepsilon_{1} D-M_{0}-1}|b|^{\varepsilon_{1} D+m(s+1) M_{0}+1}\right)^{m-1} \\
= & |b|^{D\left(m g+(m-1)\left(\eta\left(m-\varepsilon_{0}-M_{0} / D-1 / D\right)+\varepsilon_{1}+m(s+1) M_{0} / D+1 / D\right)\right)}
\end{aligned}
$$

From the inequalities (3.1.21), one finds that $D_{i}$ are increasing as functions of $H_{i}$. Namely, the value $D_{i}$ is maximal for $H_{i}=H$. Therefore, for the validity of (3.1.25) it suffices to show

$$
\begin{aligned}
\log H \leq(D(1-\eta)-D(g & +\left(m-1-\varepsilon_{1}\right) \eta+\varepsilon_{1} \\
& \left.\left.+((m-1)(s+1)-\eta) \frac{M_{0}}{D}\right)\right) \log |b|
\end{aligned}
$$

after replacing $D_{i}$ in (3.1.21) by $D$. Consequently, if there exists an integer $D$ satisfying (3.1.26) and (3.1.27), then Theorem 3.1 holds. We recall that $\eta$ defined by (3.1.20) satisfies

$$
|b|^{\eta}=|a| .
$$

Assume that

$$
\eta \leq 11(s+1) \varepsilon
$$

When

$$
\varepsilon<\min \left(\frac{1}{14 m(s+1)}, \frac{3}{44}\right) \quad \text { and } \quad D>\frac{2}{\varepsilon},
$$

one has $0 \leq g<\varepsilon / 2$ and $0<\varepsilon_{1}<3 \varepsilon / 2$. Then it follows that

$$
\begin{aligned}
& D\left(m g+(m-1)\left(\eta\left(m-\varepsilon_{1}-\frac{M_{0}}{D}-\frac{1}{D}\right)+\varepsilon_{1}+m(s+1) \frac{M_{0}}{D}+\frac{1}{D}\right)\right) \\
& \quad \leq D\left(\frac{1}{2} \varepsilon m+(m-1)\left(11 \varepsilon m(s+1)+\frac{3}{2} \varepsilon+2 \varepsilon m(s+1)+\frac{1}{2} \varepsilon\right)\right) \\
& \quad \leq D\left(\frac{1}{2} \varepsilon m+14 \varepsilon m(m-1)(s+1)\right) \leq 15 \varepsilon m(m-1)(s+1) D
\end{aligned}
$$

and

$$
\begin{aligned}
& D(1-\eta)-D\left(g+\left(m-1+\varepsilon_{1}\right) \eta+\varepsilon_{1}+((m-1)(s+1)-\eta) \frac{M_{0}}{D}\right) \\
& \quad \geq D\left(1-\left(\frac{1}{2} \varepsilon+1\right) \varepsilon\left(m+\frac{3}{2} \varepsilon\right)(s+1)+\frac{3}{2} \varepsilon+2 \varepsilon(m-1)(s+1)\right) \\
& \quad \geq D(1-\varepsilon(2+13 m(s+1))) \geq D(1-14 \varepsilon m(s+1)) .
\end{aligned}
$$

Now we put

$$
\varepsilon_{0}:=22 m(m-1)(s+1) \varepsilon .
$$


Then to show (3.1.26) it is enough to prove

$$
H^{\varepsilon_{0}} \geq|b|^{15 \varepsilon m(m-1)(s+1) D}=|b|^{\frac{15}{22} \varepsilon_{0} D},
$$

or equivalently,

$$
D \leq \frac{22 \log H}{15 \log |b|}
$$

by (3.1.30) and (3.1.32); also, to show (3.1.27) it is enough to prove

$$
H \leq|b|^{D(1-14 \varepsilon m(s+1))},
$$

or equivalently,

$$
D \geq \frac{\log H}{(1-14 \varepsilon m(s+1)) \log |b|}
$$

by (3.1.31). From the assumption $0<\varepsilon_{0}<1 / 2$, that is, $0<14 \varepsilon m(s+1)<$ $7 / 22$, it follows that

$$
\left(\frac{22}{15}-\frac{1}{1-14 \varepsilon m(s+1)}\right) \frac{\log H}{\log |b|}>1
$$

for any large $H$. This implies that there exists a positive integer $D$ which satisfies (3.1.33) and (3.1.34). From (3.1.29) and (3.1.32), one has

$$
\eta \leq \frac{\varepsilon_{0}}{2 m(m-1)} .
$$

This means $|b|^{\varepsilon_{0}} \geq|a|^{2 m(m-1)}$. If $|r|$ is sufficiently small, then $r u(r) \neq 0$. Consequently, when we choose the constant $C_{8}$ as above, depending on $u$, for any $\varepsilon_{0}$ with $0<\varepsilon_{0}<1 / 2$, if

$$
|b|^{\varepsilon_{0}} \geq C_{8}|a|^{2 m(m-1)},
$$

then

$$
\left|L_{(1,1)}\right|>\frac{H^{1-\varepsilon_{0}}}{H_{1}^{m_{1}} \cdots H_{n}^{m_{n}}}
$$

Remark 3.2. For $\varepsilon_{0}$ sufficiently small, one can improve the inequality (3.1.36) slightly.

We obtain Theorem 3.1 using the fact that the coefficient in the differential equation (3.1.1) is a block diagonal matrix, but this restriction is not necessary. One finds the bounds for the following differential equation in place of (3.1.1) in a similar way under the additional assumption that $D_{1} \geq \ldots \geq D_{n}$. 
TheOREM 3.3. Let $A_{(i)} \in M_{m_{i}}(\mathbb{Q}(x)), m=\sum_{i=1}^{n} m_{i}$ and let $\Lambda:=$ $\left\{(i, j) \mid i=1, \ldots, n ; j=1, \ldots, m_{i}\right\}$. Suppose that $f_{(i, j)} \in \mathbb{Q}[[x]]$ for $(i, j) \in$ $\Lambda$ are $G$-functions which satisfy

$$
\frac{d}{d x}\left(\begin{array}{c}
f_{(1,1)} \\
\vdots \\
f_{\left(1, m_{1}\right)} \\
f_{(2,1)} \\
\vdots \\
f_{\left(n, m_{n}\right)}
\end{array}\right)=A\left(\begin{array}{c}
f_{(1,1)} \\
\vdots \\
f_{\left(1, m_{1}\right)} \\
f_{(2,1)} \\
\vdots \\
f_{\left(n, m_{n}\right)}
\end{array}\right)
$$

where

$$
A=\left(\begin{array}{ccc}
A_{(1)} & & 0 \\
& \ddots & \\
* & & A_{(n)}
\end{array}\right) .
$$

Furthermore, assume that $f_{(1,1)}, \ldots, f_{\left(n, m_{n}\right)}$ are linearly independent over $\mathbb{Q}(x)$. Let $r=a / b \in \mathbb{Q}, a, b \in \mathbb{Z}, r \neq 0$ and $0<\varepsilon_{0}<1 / 2$ be a fixed real number. Then there exist an effective constant $C_{10}$, depending only on $f_{(i, j)}, \varepsilon_{0}, m, A$, and an effective constant $C_{11}$, depending only on $f_{(i, j)}, \varepsilon_{0}$, $m, r, A$, such that if

$$
|b|^{\varepsilon_{0}}>C_{10}|a|^{2 m(m+1)},
$$

then for any $H_{i, j} \in \mathbb{Z}$ such that $H:=\max _{(i, j) \in \Lambda}\left|H_{i, j}\right|>C_{11}$, and for $H_{i}:=\max _{1 \leq j \leq m_{i}}\left(\left|H_{i, j}\right|, 1\right)$ satisfying $H_{1} \geq \ldots \geq H_{n}>0$,

$$
\left|\sum_{(i, j) \in \Lambda} H_{i, j} f_{(i, j)}(r)\right|>\frac{H^{1-\varepsilon_{0}}}{H_{1}^{m_{1}} \cdots H_{n}^{m_{n}}} \text {. }
$$

One can apply Theorem 3.3 into some concrete $G$-functions such as the logarithm and polylogarithms.

\section{References}

[1] Y. André, G-functions and Geometry, Max-Planck-Institut, Bonn, 1989.

[2] A. Baker, Transcendental Number Theory, Cambridge University Press, Cambridge, 1975.

[3] E. Bombieri, On G-functions, in: Recent Progress in Analytic Number Theory 2, Academic Press, New York, 1981, 1-67.

[4] D. V. Chudnovsky and G. V. Chudnovsky, Applications of Padé approximations to diophantine inequalities in values of $G$-functions, in: Lecture Notes in Math. 1135, Springer, Berlin, 1985, 9-51.

[5] G. V. Chudnovsky, On some applications of diophantine approximations, Proc. Nat. Acad. Sci. U.S.A. 81 (1984), 1926-1930. 
[6] A. I. Galochkin, Estimates from below of polynomials in the values of analytic functions of a certain class, Mat. Sb. 95 (137) (1974), 396-417 (in Russian); English transl.: Math. USSR-Sb. 24 (1974), 385-407.

[7] A. B. Shidlovskiǔ, Transcendental Numbers, Walter de Gruyter, Berlin, 1989.

[8] C. L. Siegel, Über einige Anwendungen diophantischer Approximationen, Abh. Preuss. Akad. Wiss., Phys. Math. Kl. nr. 1 (1929).

[9] K. Väänänen, On linear forms of a certain class of $G$-functions and p-adic $G$ functions, Acta Arith. 36 (1980), 273-295.

TOKYO INSTITUTE OF TECHNOLOGY

MEGUROKU

OH-OKAYAMA

TOKYO 152, JAPAN

E-mail: NAGATA@MATH.TITECH.AC.JP

Received on 22.6.1992

and in revised form on 29.8.1994 